ISSN 0103-9954

\title{
ESTRUTURA E RELAÇÕES FLORÍSTICAS DE UM FRAGMENTO DE FLORESTA ESTACIONAL SEMIDECIDUAL, LONDRINA, PARANÁ, BRASIL
}

\author{
STRUCTURE AND FLORISTIC RELATIONSHIPS OF REMNANT FOREST, LONDRINA, PARANÁ \\ STATE, BRAZIL
}

\author{
Daniela Aparecida Estevan ${ }^{1}$ Ana Odete Santos Vieira ${ }^{2}$ Maurício Romero Gorenstein ${ }^{3}$
}

\begin{abstract}
RESUMO
O maior fragmento da Fazenda Figueira (Londrina-PR) com área de 266 ha, é uma Reserva Particular de Patrimônio Natural, e um dos mais importantes remanescentes da região. $\mathrm{O}$ objetivo deste trabalho foi caracterizar a estrutura e a diversidade arbórea do referido fragmento, e avaliar a relação florística com outros fragmentos de Floresta Estacional Semidecidual estudados no estado do Paraná. Foram alocadas 100 parcelas de $10 \times 10 \mathrm{~m}$, e em cada uma delas foram amostrados os indivíduos arbóreos com $15 \mathrm{~cm}$ ou mais de Circunferência a Altura do Peito, resultando em 1569 indivíduos, distribuídos em 32 famílias, 68 gêneros e 93 espécies. As famílias que mais se destacaram em número de espécies foram Fabaceae (16 espécies), Meliaceae (nove espécies) e Myrtaceae (nove espécies). Trichilia clausseni C.DC. foi a espécie de maior valor de importância no fragmento estudado, reflexo dos maiores valores de densidade e frequência relativa. A maior dominância relativa foi apresentada por Gallesia integrifolia (Spreng.) Harms, por apresentar indivíduos de grande porte com os maiores valores de área basal. O índice de diversidade de Shannon encontrado foi de 3,37 nats/ind, valor este considerado expressivo e similar a outros levantamentos na região, e o índice de equabilidade de Pielou foi de 0,743 indicando equilíbrio no número de indivíduos entre as espécies. A presença de espécies com apenas um indivíduo amostrado e de espécies consideradas raras no Paraná, bem como a diversidade total das espécies e o grau de conservação do fragmento, demonstram a importância da preservação e manejo do remanescente. A proximidade geográfica associada a fatores ambientais semelhantes, assim como ao tipo de unidade fitogeográfica predominante, evidenciaram relações florísticas mais próximas, por outro lado, fatores ambientais locais como proximidade a cursos d'água diferenciaram a flora de locais próximos.
\end{abstract}

Palavras-chave: fitossociologia; Mata Atlântica; bacia do rio Tibagi; sul do Brasil.

\section{ABSTRACT}

The largest fragment of Figueira Farm (Londrina-PR state) with an area of 266 ha, is a Private Natural Heritage Reserve, and one of the most important remnants of the region. The aim of this study was to characterize the structure and tree diversity of this fragment, and evaluate the floristic relationship with other fragments of semideciduous forest studied in the state of Paraná. One hundred plots of $10 \times 10 \mathrm{~m}$ were allocated and the arboreal individuals with $15 \mathrm{~cm}$ or more in circumference at breast height were sampled, resulting in 1,569 individuals, 32 families, 66 genera and 93 species. Families with greater number of species were Fabaceae (16 species), Meliaceae (nine species) and Myrtaceae (nine species). Trichilia clausseni C.DC. was the species with the highest importance in the study, reflecting higher values of density and relative frequency in the fragment. The largest relative dominance was presented by Gallesia integrifolia (Spreng.) Harms, by

1 Bióloga, Dra ${ }^{\mathrm{a}}$, Professora da Coordenação de Ciências Biológicas, Universidade Tecnológica Federal do Paraná, Campus Dois Vizinhos, CEP 85660-000, Dois Vizinhos (PR), Brasil. danielaaestevan@utfpr.edu.br

2 Bióloga, $\mathrm{Dr}^{\mathrm{a}}$., Professora do Departamento de Biologia Animal e Vegetal, Centro de Ciências Biológicas, Universidade Estadual de Londrina, Caixa Postal 10.011, CEP 86057-970, Londrina (PR), Brasil. aovieira@uel.br

3 Engenheiro Florestal, Dr., Professor da Coordenação de Engenharia Florestal, Universidade Tecnológica Federal do Paraná, Campus Dois Vizinhos, CEP 85660-000, Dois Vizinhos (PR), Brasil. mauriciorg@utfpr.edu.br

Recebido para publicação em 24/07/2013 e aceito em 22/09/2014

Ci. Fl., v. 26, n. 3, jul.-set., 2016 
presenting large individuals with the highest values of basal area. The Shannon diversity index was found to be 3.37 nats/ind, a value considered significant and similar to other surveys in the region, and the index of evenness by Pielou was 0.743 , indicating balance in the number of individuals among species. The presence of singletons and rare tree species in Paraná as well as the total diversity of species and the degree of conservation of the fragment, demonstrate the importance of the preservation and management of the remnant. Geographical proximity associated with similar environment factors as well as the predominant type of phytogeographic unit, showed closer floristic relationships, on the other hand, local environmental factors such as proximity to watercourses differentiated its flora of nearby places.

Keywords: phytosociology; Atlantic Forest; Tibagy river basin; southern Brazil.

\section{INTRODUÇÃO}

O Estado do Paraná com uma área total de $199.575 \mathrm{~km}^{2}$ contava no início do século com $83,4 \%$ de sua área coberta por florestas nativas, componentes do bioma Mata Atlântica, que atualmente restringiu-se a 5,2\% (LOUREIRO, 2004). A Floresta Estacional Semidecidual é a segunda principal formação florestal deste bioma e também a mais ameaçada, cobrindo originalmente $37,3 \%$ do estado, mas restando atualmente apenas $3,4 \%$ do total (CAMPOS e SILVEIRA-FILHO, 2010). Diversos fatores relacionados com a exploração das riquezas da terra e sua consequente colonização contribuíram para a diminuição da área florestada do estado.

A Floresta Estacional Semidecidual apresenta como característica marcante, a perda de folhas de $20-50 \%$ das árvores do dossel durante a estação mais seca e fria do ano. No Paraná, tais florestas ocorrem em altitudes entre 200 e $800 \mathrm{~m}$, no Oeste, Noroeste e Norte do Estado, em grande parte coincidindo com a região de ocorrência da vulgarmente conhecida "terra roxa" (CAMPOS e SILVEIRA-FILHO, 2010; RODERJAN et al., 2002). Este tipo de formação florestal caracterizase por árvores que atingem até 30 metros de altura, sem formar cobertura superior contínua ("árvores emergentes"), cujos troncos são grossos e de fuste longo (IBGE, 1992).

O município de Londrina, um dos municípios pertencentes à bacia hidrográfica do rio Tibagi (Paraná), está localizado no segundo planalto paranaense e apresenta como tipo de vegetação dominante a Floresta Estacional Semidecidual (DIAS et al., 2002). A fazenda Figueira localizada neste município, foi doada pelo ex-aluno Alexandre von Pritzelwitz à Escola Superior de Agricultura "Luiz de Queiroz" no ano de 2000, sendo transformada no mesmo ano em Estação Experimental Agrozootécnica Hildegard
Georgina von Pritzelwitz. Esta estação apresenta um dos maiores remanescentes florestais da região, constituída por uma área de 1.126,1 ha de mata natural, distribuída em fragmentos, transformados em Reserva Particular de Patrimônio Natural (Portaria ${ }^{\circ}$ 195/98/IAP/GP).

Os remanescentes vegetacionais podem ser amostras pouco representativas da vegetação original, devido aos efeitos da fragmentação. Entretanto, o conhecimento sobre a diversidade e a organização espacial das suas comunidades vegetais poderá gerar subsídios para o manejo e a conservação a longo prazo (MACHADO et al, 2008).

A Floresta Estacional Semidecidual no Paraná vem sendo estudada desde o início da década de 90 por vários autores que contribuem com dados sobre a sua flora e a estrutura (BIANCHINI et al., 2003; COSTA et al., 2011; FERREIRA Jr., 2009; KILMA et al., 2012; MARESTONI, 2007; MORESCHI e SAMPAIO, 2008; SOUZA e MONTEIRO, 2005; SOUZA et al., 2011; PEZZATO, 2004; SÁ, 2004; SAKURAGUI e JANUNZZI, 2009; SILVA et al., 1995; SOARESSILVA et al., 1992; SOARES-SILVA et al., 1998; VEIGA et al., 2003; VIANI et al., 2011).

Os levantamentos fitossociológicos geram dados qualitativos e quantitativos que permitem fazer a avaliação momentânea da estrutura e o grau de conservação da vegetação. Sendo assim, os objetivos deste trabalho foram: I) caracterizar a estrutura e a diversidade arbórea do maior fragmento da RPPN da fazenda Figueira, Londrina - PR; (II) avaliar a relação florística deste fragmento com outros fragmentos de Floresta Estacional Semidecidual estudados no estado do Paraná.

\section{MATERIAL E MÉTODOS}

A Fazenda Figueira está situada no 
município de Londrina, região mais baixa e próxima à foz da bacia hidrográfica do rio Tibagi, no terceiro planalto paranaense. A propriedade, com uma área total de aproximadamente 3.700 ha, é margeada pelos rios Tibagi (divisa do município) a leste e Taquara a norte e nela são desenvolvidas atividades agropecuárias.

A área total de Floresta Estacional Semidecidual (FES) é de 1.126,1 ha, correspondendo a mais de $20 \%$ da área total da fazenda, e apesar da vegetação estar dividida em 33 fragmentos e apresentar certo grau de perturbação, representa uma das principais áreas de remanescentes naturais do município, transformada em Reserva Particular do Patrimônio Natural - RPPN Mata do Barão em 1998. O fragmento escolhido para este estudo foi o maior ocupando uma área de 266 ha.

A formação geológica da região é a Formação Serra Geral, pertencente ao Grupo São Bento, e se caracteriza por erupções de rochas básicas, principalmente o basalto (PINESE, 2002). Os tipos de solos predominantes são os neossolos, nitrossolos, e latossolos vermelhos escuros (EMBRAPA-IAPAR, 1984), solos estes que se destacam por sua grande fertilidade natural (STIPP, 2002). As altitudes na região não ultrapassam 800 m, e o clima é do tipo Cfa (segundo a classificação de Koeppen), ou seja, subtropical úmido com verão quente, com a média térmica anual de $21^{\circ} \mathrm{C}$, média máxima de $28^{\circ} \mathrm{C}$, média mínima de $16^{\circ} \mathrm{C}$ e média pluviométrica de $1600 \mathrm{~mm}$ (DANNI-OLIVEIRA et al., 2002).

Para a amostragem fitossociológica foram alocadas 100 parcelas de $10 \times 10 \mathrm{~m}$, totalizando uma área de $10.000 \mathrm{~m}^{2}$, que corresponde a cerca de $0,37 \%$ da área total do fragmento. Os indivíduos amostrados foram incluídos segundo o critério de circunferência a altura do peito (CAP) igual ou superior a $15 \mathrm{~cm}$. Cada indivíduo amostrado foi marcado com plaquetas numeradas, e teve anotado em fichas de campo sua circunferência a altura do peito (CAP) e sua altura total estimada visualmente, tendo por base a altura máxima da tesoura de poda alta com cabo telescópio utilizado para coleta de material testemunho.

Todo o material botânico coletado foi prensado, seco e herborizado segundo os métodos usuais de herborização (JUDD et al., 2009), no herbário da Universidade Estadual de Londrina (FUEL) onde estão depositados os materiais testemunhos. Para a identificação do material, utilizaram-se bibliografias especializadas, comparações com materiais incorporados ao herbário (FUEL) e o auxílio de especialistas em famílias de angiospermas. Para a delimitação das famílias utilizou-se o sistema de classificação do Angiosperm Phylogeny Group (APG III, 2009).

Os descritores fitossociológicos: densidade, frequência, dominância e importância (IVI) foram estimados como descritos por Martins (1993), por meio do conjunto de programas FITOPAC, de autoria de George J. Shepherd, da Universidade Estadual de Campinas. Para avaliação da diversidade foi utilizado o índice de Shannon e da equabilidade o índice de Pielou (MAGURRAN, 1988).

Para o estudo das relações florísticas entre o fragmento estudado e outros remanescentes de FES ou FES ecótono com Floresta Ombrófila Mista (FOM) do estado do Paraná foram utilizados 19 levantamentos, sendo 12 em regiões de FES e sete em áreas de ecótono entre FES e FOM (Tabela 1). $\mathrm{O}$ banco de dados consistiu de uma matriz binária de presença e ausência de espécies, tendo sido consideradas apenas as espécies com binômio completo, ou seja, foram excluídas identificações em nível de famílias e gêneros. Calculou-se o índice de dissimilaridade de Jaccard (KREBS, 1999) como medida de distância e foi feita uma análise de agrupamentos através do método de ligação completa. Este método foi escolhido porque apresentou maior valor de coeficiente de correlação cofenético. Para essas análises utilizou-se o pacote "Vegan" do programa estatístico R (R Development Core Team, 2010).

\section{RESULTADOS E DISCUSSÃO}

Foi amostrado cerca de $0,37 \%$ da área total do fragmento maior da RPPN, em 100 parcelas, sendo registrados 1.560 indivíduos/ha, distribuídos em 32 famílias, 68 gêneros e 92 espécies (Tabela 2). Destas espécies, uma espécie de Myrtaceae ficou sem identificação em nível de gênero.

O número médio de indivíduos encontrados por parcelas foi de 15,6. A área basal total foi de $50,17 \mathrm{~m}^{2} / \mathrm{ha}$, valor considerado relativamente alto, podendo enquadrar o fragmento em estágio sucessional avançado segundo a resolução do CONAMA n ${ }^{\circ}$ 2, de 18 de março de 1994. O índice de equabilidade obtido foi de $\mathrm{J}=0,743$, indicando que as espécies ocorrentes apresentam uma boa distribuição de abundância, com exceção da espécie Trichilia clausseni que apresentou uma densidade relativa alta na área (Tabela 2). O índice 
TABELA 1: Listagem dos levantamentos florísticos e fitossociológicos utilizados para a comparação com este estudo.

TABLE 1: Phytosociological and floristic studies used to compare with the present research.

\begin{tabular}{|c|c|c|c|c|}
\hline CÓD & Município & Fitofisionomia & Número de espécies & Fonte \\
\hline ASTOR & Astorga & FES & 63 & Veiga et al., 2003 \\
\hline CAPLEO & Capitão Leônidas Marques & FES/FOM & 76 & Pezzatto, 2004 \\
\hline DIANOR & Diamante do Norte & FES & 136 & Sakuragui e Janunzzi, 2009 \\
\hline DOISVIZ & Dois Vizinhos & FES/FOM & 96 & Souza et al., 2011 \\
\hline IBI1 & Ibiporã & FES & 93 & Costa et al., 2011 \\
\hline IBI2 & Ibiporã & FES & 73 & Soares-Silva et al., 1992 \\
\hline LOND1 & Londrina & FES & 92 & Este trabalho \\
\hline LOND2 & Londrina & FES & 96 & Soares e Silva, 1998 \\
\hline LOND3 & Londrina & FES & 64 & Bianchini et al., 2003 \\
\hline LOND4 & Londrina & FES & 197 & Rosseto e Vieira, 2013 \\
\hline LUP & Lupionópolis & FES & 94 & Marestoni, 2007 \\
\hline MARIN & Maringá & FES & 66 & Moreschi e Sampaio, 2008 \\
\hline MAUA & Mauá da Serra & FES/FOM & 53 & Ferreira Jr., 2009 \\
\hline PORTRIC & Porto Rico & FES & 85 & Souza e Monteiro, 2005 \\
\hline QUEDIGU1 & Quedas do Iguaçu & FES/FOM & 128 & Viani et al., 2011 \\
\hline QUEDIGU2 & Quedas do Iguaçu & FES/FOM & 98 & Kilma et al., 2012 \\
\hline SAOJER & São Jerônimo da Serra & FES/FOM & 178 & Sá, 2004 \\
\hline SAPOP & Sapopema & *FES/FOM & 125 & Silva et al., 1995 \\
\hline TAM & Tamarana & FES/FOM & 57 & Ferreira Jr., 2009 \\
\hline
\end{tabular}

Em que: CÓD = código da área utilizado na Figura 1; FES - Floresta Estacional Semidecidual e FES/FOM = área de ecótono entre Floresta Estacional Semidecidual e Floresta Ombrófila Mista. O tipo de vegetação foi definido pelos próprios autores. * classificação não fornecida pelos autores e obtida a partir do mapa ITCG (2009).

de diversidade de Shannon encontrado foi de 3,374 nats/ind., valor similar ao encontrado para outros levantamentos na região sul e sudeste. Para os trabalhos de FES e ecótono FES/FOM no Paraná, os valores de diversidade de Shannon variaram entre 3,31 e 3,9 (BIANCHINI et al., 2003; FERREIRA Jr., 2009; SOARES-SILVA et al., 1992; SOARESSILVA et al., 1998; VIANI et al., 2011), e para florestas estacionais semidecíduas no Brasil ficam entre 3,16 a 4,29 segundo Leitão Filho (1987). Entretanto, deve-se lembrar que a comparação entre estes estudos não é rigorosa, pois cada um deles reflete um fragmento com diferentes históricos de perturbação, avaliados sob diferentes métodos de amostragem e critérios de inclusão.

As quatro famílias com maior número de espécies em ordem decrescente foram Fabaceae (17 espécies), Meliaceae (nove espécies), Myrtaceae (oito espécies) e Lauraceae (sete espécies), representado juntas $44,08 \%$ do total de espécies. Essas famílias também se destacaram nas FES e nos ecótonos FES e FOM dos trabalhos utilizados na análise de similaridade e estão entre as famílias mais importantes da bacia do rio Tibagi (DIAS et al., 2002). Estas famílias também apresentaram alta riqueza em estudo realizado em dez fragmentos de FES no planalto Meridional, do Rio Grande do Sul (KILCA e LONGHI, 2011), bem como quando se considera a "floresta Atlântica" no sudeste do Brasil (OLIVEIRA-FILHO e FONTES, 2000).

As famílias Moraceae (quatro espécies) e Phytolaccaceae (duas espécies), apesar de apresentarem poucas espécies, se destacaram em valor de importância, devido principalmente ao grande porte das espécies amostradas. Os dados também corroboram os resultados sobre famílias de maior valor de importância na região do baixo Tibagi (DIAS et al., 2002).

A Tabela 2 apresenta as espécies amostradas no levantamento e seus respectivos descritores fitossociológicos, ordenados segundo valores decrescentes de VI. Além das espécies 
TABELA 2: Relação das espécies amostradas no maior fragmento de Floresta Estacional Semidecidual (Londrina-PR) e seus respectivos parâmetros fitossociológicos.

TABLE 2: Tree species sampled in the largest remnant of Seasonal Semideciduous Forest (Londrina-PR state) and phytosociological parameters.

\begin{tabular}{|c|c|c|c|c|c|c|c|c|}
\hline Espécie & Família & $\begin{array}{l}\mathrm{N}^{\circ} \\
\text { ind }\end{array}$ & DR & DoR & FR & IVI & IVC & $\begin{array}{l}\mathrm{N}^{\circ} \text {. do } \\
\text { coletor }\end{array}$ \\
\hline Trichilia clausseni C.DC. & Meliaceae & 353 & 22,62 & 6,30 & 9,02 & 12,65 & 14,46 & DAE 331 \\
\hline $\begin{array}{l}\text { Gallesia integrifolia (Spreng.) } \\
\text { Harms. }\end{array}$ & Phytolaccaceae & 55 & 3,53 & 24,48 & 4,35 & 10,79 & 14,01 & DAE 345 \\
\hline Cabralea canjerana (Vell.) Mart. & Meliaceae & 74 & 4,74 & 6,90 & 5,08 & 5,57 & 5,82 & DAE 325 \\
\hline $\begin{array}{l}\text { Actinostemon concolor (Spreng.) } \\
\text { Müll. Arg. }\end{array}$ & Euphorbiaceae & 126 & 8,07 & 1,00 & 4,97 & 4,68 & 4,53 & DAE 291 \\
\hline Ficus guaranitica Chodat & Moraceae & 4 & 0,25 & 12,23 & 0,41 & 4,30 & 6,24 & MCL 110 \\
\hline $\begin{array}{l}\text { Nectandra megapotamica } \\
\text { (Spreng.) Mez }\end{array}$ & Lauraceae & 65 & 4,16 & 3,72 & 4,66 & 4,18 & 3,94 & DAE 296 \\
\hline $\begin{array}{l}\text { Sorocea bonplandii (Baill.) W.C. } \\
\text { Burger et al. }\end{array}$ & Moraceae & 99 & 6,34 & 0,89 & 5,08 & 4,10 & 3,61 & DAE 335 \\
\hline Euterpe edulis Mart. & Arecaceae & 68 & 4,35 & 1,51 & 4,3 & 3,39 & 2,93 & MCL 68 \\
\hline Guarea kunthiana A Juss. & Meliaceae & 64 & 4,10 & 1,02 & 4,87 & 3,33 & 2,56 & DAE 327 \\
\hline Achatocarpus praecox Griseb. & Achatocarpaceae & 11 & 0,70 & 7,47 & 0,83 & 3,00 & 4,09 & DAE 279 \\
\hline Trichilia catigua A Juss. & Meliaceae & 52 & 3,33 & 0,53 & 4,04 & 2,63 & 1,93 & DAE 330 \\
\hline $\begin{array}{l}\text { Aspidosperma polyneuron Müll. } \\
\text { Arg. }\end{array}$ & Apocynaceae & 20 & 1,28 & 4,65 & 1,76 & 2,56 & 2,96 & DAE 283 \\
\hline Bongainvillia spectabilis Willd. & Nyctaginaceae & 26 & 1,67 & 3,10 & 2,38 & 2,38 & 2,39 & DAE 342 \\
\hline $\begin{array}{l}\text { Endlicheria paniculata (Spreng.) } \\
\text { J.F. Macbr. }\end{array}$ & Lauraceae & 42 & 2,69 & 0,58 & 3,73 & 2,34 & 1,64 & MCL 88 \\
\hline Holocalyx balansae Micheli & Fabaceae & 25 & 1,60 & 2,38 & 1,97 & 1,98 & 1,99 & DAE 313 \\
\hline Picramnia ramiflora Planch. & Picramniaceae & 41 & 2,62 & 0,31 & 2,49 & 1,81 & 1,47 & DAE 347 \\
\hline $\begin{array}{l}\text { Pouteria beaurepairei (Glaz. \& } \\
\text { Raunk.) Baehni }\end{array}$ & Sapotaceae & 29 & 1,86 & 0,73 & 2,28 & 1,62 & 1,29 & DAE 364 \\
\hline $\begin{array}{l}\text { Chrysophyllum gonocarpum } \\
\text { (Mart. \& Eichl ex Miq.) Engl. }\end{array}$ & Sapotaceae & 22 & 1,41 & 1,41 & 1,87 & 1,56 & 1,41 & DAE 362 \\
\hline Eugenia ramboi D. Legrand & Myrtaceae & 24 & 1,54 & 0,71 & 2,38 & 1,54 & 1,12 & DAE 338 \\
\hline Ocotea indecora (Schott.) Mez & Lauraceae & 25 & 1,60 & 0,89 & 1,76 & 1,41 & 1,24 & DAE 300 \\
\hline Pilocarpus pennatifolius Lem. & Rutaceae & 28 & 1,79 & 0,19 & 1,87 & 1,28 & 0,99 & DAE 353 \\
\hline Inga marginata Willd. & Fabaceae & 19 & 1,22 & 0,32 & 1,97 & 1,17 & 0,77 & DAE 308 \\
\hline $\begin{array}{l}\text { Balfourodendron riedelianum } \\
\text { (Engl.) Engl. }\end{array}$ & Rutaceae & 16 & 1,03 & 0,63 & 1,55 & 1,07 & 0,83 & DAE 352 \\
\hline $\begin{array}{l}\text { Muellera campestris (Mart. ex } \\
\text { Benth.) M.J. Silva \& A.M.G. } \\
\text { Azevedo }\end{array}$ & Fabaceae & 11 & 0,70 & 1,01 & 1,14 & 0,95 & 0,86 & MCL 101 \\
\hline $\begin{array}{l}\text { Campomanesia } \quad \text { xanthocarpa } \\
\text { (Mart.) O. Berg }\end{array}$ & Myrtaceae & 15 & 0,96 & 0,18 & 1,24 & 0,80 & 0,57 & DAE 306 \\
\hline Ficus insipida Wild. & Moraceae & 5 & 0,32 & 1,48 & 0,52 & 0,77 & 0,90 & MCL 111 \\
\hline Cedrela fissilis Vell. & Meliaceae & 10 & 0,64 & 0,48 & 1,14 & 0,76 & 0,56 & DAE 326 \\
\hline
\end{tabular}


TABELA 2: Continuação...

TABLE 2: Continued...

\begin{tabular}{|c|c|c|c|c|c|c|c|c|}
\hline Espécie & Família & $\begin{array}{l}\mathrm{N}^{\circ} . \\
\text { ind }\end{array}$ & DR & DoR & FR & IVI & IVC & $\begin{array}{l}\mathrm{N}^{\circ} \text {. do } \\
\text { coletor }\end{array}$ \\
\hline $\begin{array}{l}\text { Schizolobium parahyba (Vell.) } \\
\text { Blake }\end{array}$ & Fabaceae & 3 & 0,19 & 1,73 & 0,31 & 0,74 & 0,96 & MCL 94 \\
\hline $\begin{array}{l}\text { Syagrus romanzoffiana (Cham.) } \\
\text { Glassman }\end{array}$ & Arecaceae & 9 & 0,57 & 0,47 & 1,04 & 0,70 & 0,52 & MCL 69 \\
\hline $\begin{array}{l}\text { Machaerium stipitatum (DC.) } \\
\text { Vogel }\end{array}$ & Fabaceae & 11 & 0,70 & 0,17 & 1,14 & 0,67 & 0,44 & DAE 319 \\
\hline Trichilia pallida $\mathrm{Sw}$. & Meliaceae & 11 & 0,70 & 0,12 & 1,04 & 0,62 & 0,41 & DAE 334 \\
\hline Eugenia florida DC. & Myrtaceae & 9 & 0,71 & 0,07 & 0,83 & 0,54 & 0,39 & DAE 337 \\
\hline Ocotea puberula (Rich.) Nees & Lauraceae & 6 & 0,38 & 0,59 & 0,62 & 0,53 & 0,49 & DAE 301 \\
\hline Alchornea sp. & Euphorbiaceae & 6 & 0,38 & 0,59 & 0,52 & 0,50 & 0,49 & DAE 292 \\
\hline Phytolacca dioica $\mathrm{L}$. & Phytolaccaceae & 1 & 0,06 & 1,31 & 0,1 & 0,49 & 0,68 & DAE 346 \\
\hline Astronium graveolens Jacq. & Anacardiaceae & 8 & 0,51 & 0,12 & 0,83 & 0,49 & 0,32 & DAE 280 \\
\hline Solanum argenteum Dunal & Solanaceae & 7 & 0,45 & 0,26 & 0,73 & 0,48 & 0,36 & MCL 153 \\
\hline Miconia discolor DC. & Melastomataceae & 10 & 0,64 & 0,06 & 0,73 & 0,48 & 0,35 & DAE 232 \\
\hline Eugenia handroi (Mattos) Mattos & Myrtaceae & 5 & 0,32 & 0,80 & 0,31 & 0,48 & 0,56 & MCL 120 \\
\hline Prockia crucis P. Bromne ex L. & Salicaceae & 7 & 0,45 & 0,13 & 0,73 & 0,44 & 0,29 & DAE 358 \\
\hline $\begin{array}{l}\text { Ceiba speciosa (A. St.-Hil.) } \\
\text { Ravenna }\end{array}$ & Malvaceae & 3 & 0,19 & 0,80 & 0,31 & 0,43 & 0,49 & MCL 106 \\
\hline $\begin{array}{l}\text { Ocotea } \text { cf. diospyrifolia (Meisn.) } \\
\text { Mez }\end{array}$ & Lauraceae & 2 & 0,13 & 0,88 & 0,21 & 0,41 & 0,50 & DAE 298 \\
\hline $\begin{array}{l}\text { Dahlstedtia floribunda (Vogel) } \\
\text { M.J. Silva \& A.M.G. Azevedo }\end{array}$ & Fabaceae & 6 & 0,38 & 0,21 & 0,62 & 0,40 & 0,30 & DAE 316 \\
\hline $\begin{array}{l}\text { Myrceugenia miersiana (Gardn.) } \\
\text { D. Lerg. \& Kaus. }\end{array}$ & Myrtaceae & 6 & 0,38 & 0,09 & 0,62 & 0,36 & 0,24 & DAE 340 \\
\hline Erythrina sp. & Fabaceae & 1 & 0,06 & 0,92 & 0,1 & 0,36 & 0,49 & DAE 312 \\
\hline Bauhinia forficata Link & Fabaceae & 5 & 0,32 & 0,19 & 0,52 & 0,34 & 0,26 & MCL 91 \\
\hline Ocotea silvestris Vattimo-Gil & Lauraceae & 6 & 0,38 & 0,13 & 0,52 & 0,34 & 0,26 & DAE 302 \\
\hline $\begin{array}{l}\text { Senegalia velutina (DC.) Seigler } \\
\text { \& Ebinger }\end{array}$ & Fabaceae & 5 & 0,32 & 0,29 & 0,41 & 0,34 & 0,31 & DAE 307 \\
\hline $\begin{array}{l}\text { Machaerium paraguariense } \\
\text { Hassl. }\end{array}$ & Fabaceae & 5 & 0,32 & 0,17 & 0,52 & 0,34 & 0,25 & DAE 317 \\
\hline Myrtaceae sp. & Myrtaceae & 5 & 0,32 & 0,16 & 0,52 & 0,33 & 0,24 & \\
\hline $\begin{array}{l}\text { Aegiphila integrifolia (Jacq.) } \\
\text { Moldenke }\end{array}$ & Lamiaceae & 7 & 0,45 & 0,12 & 0,41 & 0,33 & 0,29 & DAE 368 \\
\hline $\begin{array}{l}\text { Schefflera angustissima } \\
\text { (Marchal) Frodin }\end{array}$ & Araliaceae & 3 & 0,19 & 0,44 & 0,31 & 0,31 & 0,32 & MCL 67 \\
\hline $\begin{array}{l}\text { Cariniana estrellensis (Raddi) } \\
\text { Kuntze }\end{array}$ & Lecythidaceae & 2 & 0,13 & 0,57 & 0,21 & 0,30 & 0,35 & DAE 303 \\
\hline Pisonia aculeata $\mathrm{L}$. & Nyctaginaceae & 4 & 0,25 & 0,33 & 0,31 & 0,30 & 0,29 & DAE 344 \\
\hline $\begin{array}{l}\text { Piptadenia gonoacantha (Mart.) } \\
\text { J.F.Macbr. }\end{array}$ & abaceae & 2 & 0,13 & 0,52 & 0,21 & 0,29 & 0,33 & MCL 100 \\
\hline Heliocarpus popayanensis Kunth. & Malvaceae & 3 & 0,19 & 0,31 & 0,31 & 0,27 & 0,25 & DAE 323 \\
\hline
\end{tabular}

Continua... 
TABELA 2: Continuação...

TABLE 2: Continued...

\begin{tabular}{|c|c|c|c|c|c|c|c|c|}
\hline Espécie & Família & $\begin{array}{l}\mathrm{N}^{\circ} \text {. } \\
\text { ind }\end{array}$ & DR & DoR & FR & IVI & IVC & $\begin{array}{l}\mathrm{N}^{\circ} \text {. do } \\
\text { coletor }\end{array}$ \\
\hline Piper amalago $\mathrm{L}$. & Piperaceae & 5 & 0,32 & 0,03 & 0,41 & 0,25 & 0,18 & MCL 129 \\
\hline Guarea macrophylla Vahl & Meliaceae & 5 & 0,32 & 0,03 & 0,41 & 0,25 & 0,18 & DAE 328 \\
\hline Eugenia uniflora $\mathrm{L}$. & Myrtaceae & 4 & 0,25 & 0,04 & 0,41 & 0,23 & 0,15 & DAE 339 \\
\hline Trichilia pallens C. DC. & Meliaceae & 4 & 0,25 & 0,04 & 0,41 & 0,23 & 0,15 & DAE 333 \\
\hline $\begin{array}{l}\text { Senegalia polyphylla } \\
\text { Britton \& Rose }\end{array}$ & Fabaceae & 2 & 0,13 & 0,33 & 0,21 & 0,22 & 0,23 & DAE 305 \\
\hline Ocotea elegans $\mathrm{Mez}$ & Lauraceae & 3 & 0,19 & 0,12 & 0,31 & 0,21 & 0,16 & DAE 299 \\
\hline Alseis floribunda Schott. & Rubiaceae & 3 & 0,19 & 0,11 & 0,31 & 0,20 & 0,15 & MCL 134 \\
\hline $\begin{array}{l}\text { Aralia warmingiana (March.) J. } \\
\text { Wen. }\end{array}$ & Araliaceae & 2 & 0,13 & 0,26 & 0,21 & 0,20 & 0,20 & DAE 285 \\
\hline $\begin{array}{l}\text { Dahlstedtia muehlbergiana } \\
\text { (Hassl.) M.J.Silva \& A.M.G. } \\
\text { Azevedo }\end{array}$ & 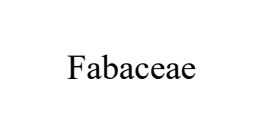 & 3 & 0,19 & 0,07 & 0,31 & 0,19 & 0,13 & DAE 315 \\
\hline Pisonia ambigua Heimerl & Nyctaginaceae & 3 & 0,19 & 0,05 & 0,31 & 0,18 & 0,12 & \\
\hline Trichilia casaretti C. DC. & Meliaceae & 3 & 0,19 & 0,03 & 0,31 & 0,18 & 0,11 & DAE 329 \\
\hline Poecilanthe parviflora Benth. & Fabaceae & 3 & 0,19 & 0,02 & 0,31 & 0,17 & 0,11 & MCL 105 \\
\hline Myrocarpus frondosus Allemão & Fabaceae & 2 & 0,13 & 0,17 & 0,21 & 0,17 & 0,15 & DAE 320 \\
\hline Guapira hirsuta (Choisy.) Lundell & Nyctaginaceae & 3 & 0,19 & 0,01 & 0,31 & 0,17 & 0,10 & DAE 343 \\
\hline $\begin{array}{l}\text { Annona sylvatica (ASt.-Hil.) } \\
\text { Mart. }\end{array}$ & Annonaceae & 2 & 0,13 & 0,14 & 0,21 & 0,16 & 0,14 & DAE 282 \\
\hline Myrsine umbelata Mart. & Primulaceae & 2 & 0,13 & 0,11 & 0,21 & 0,15 & 0,12 & DAE 336 \\
\hline $\begin{array}{l}\text { Maclura tinctoria (L.) D. Don ex } \\
\text { Steud. }\end{array}$ & Moraceae & 2 & 0,13 & 0,10 & 0,21 & 0,15 & 0,12 & MCL 113 \\
\hline Machaerium scleroxylon Tul. & Fabaceae & 2 & 0,13 & 0,04 & 0,21 & 0,13 & 0,09 & DAE 318 \\
\hline $\begin{array}{l}\text { Citronella paniculata (Mart.) } \\
\text { Howard }\end{array}$ & Icacinaceae & 2 & 0,13 & 0,04 & 0,21 & 0,13 & 0,09 & DAE 295 \\
\hline $\begin{array}{l}\text { Miconia } \quad \text { pusilliflora } \quad \text { (DC.) } \\
\text { Naudin. }\end{array}$ & Melastomataceae & 2 & 0,13 & 0,02 & 0,21 & 0,12 & 0,08 & MCL 107 \\
\hline $\begin{array}{l}\text { Aloysia virgata (Ruiz \& Pavon) } \\
\text { Juss. }\end{array}$ & Verbenaceae & 1 & 0,06 & 0,11 & 0,1 & 0,09 & 0,09 & MCL 165 \\
\hline Guazuma ulmifolia Lam & Malvaceae & 1 & 0,06 & 0,11 & 0,1 & 0,09 & 0,09 & DAE 286 \\
\hline $\begin{array}{l}\text { Chrysophyllum marginatum } \\
\text { (Hook. \& Arn.) Radlk. }\end{array}$ & Sapotaceae & 1 & 0,06 & 0,07 & 0,1 & 0,08 & 0,07 & DAE 363 \\
\hline $\begin{array}{l}\text { Bastardiopsis densiflora (Hook. } \\
\& \text { Arn.) Hassl. }\end{array}$ & Malvaceae & 1 & 0,06 & 0,07 & 0,1 & 0,08 & 0,07 & DAE 322 \\
\hline Strychnos brasiliensis Mart. & Loganiaceae & 1 & 0,06 & 0,05 & 0,1 & 0,07 & 0,06 & DAE 321 \\
\hline Ruprechtia paranensis Pendry. & Polygonaceae & 1 & 0,06 & 0,03 & 0,1 & 0,06 & 0,05 & DAE 349 \\
\hline Zanthoxylum caribaeum Lam. & Rutaceae & 1 & 0,06 & 0,03 & 0,1 & 0,06 & 0,05 & DAE 356 \\
\hline Urera caracasana (Jacq.) Griseb. & Urticaceae & 1 & 0,06 & 0,01 & 0,1 & 0,06 & 0,04 & DAE 243 \\
\hline Mollinedia clavigera Tul. & Monimiaceae & 1 & 0,06 & 0,01 & 0,1 & 0,06 & 0,04 & MCL 109 \\
\hline Solanum sp. & Solanaceae & 1 & 0,06 & 0,01 & 0,1 & 0,06 & 0,04 & \\
\hline
\end{tabular}


TABELA 2: Continuação...

TABLE 2: Continued...

\begin{tabular}{|c|c|c|c|c|c|c|c|c|}
\hline Espécie & Família & $\begin{array}{l}\mathrm{N}^{\circ} . \\
\text { ind }\end{array}$ & DR & DoR & FR & IVI & IVC & $\begin{array}{l}\mathrm{N}^{\circ} \text {. do } \\
\text { coletor }\end{array}$ \\
\hline $\begin{array}{l}\text { Myrsine laetevirens }(\mathrm{Mez}) \\
\text { Arechav. }\end{array}$ & Myrsinaceae & 1 & 0,06 & 0,01 & 0,1 & 0,06 & 0,04 & MCL 114 \\
\hline $\begin{array}{l}\text { Dahlstedtia muehlbergiana, } \\
\text { Machaerium }\end{array}$ & Fabaceae & 1 & 0,06 & 0,01 & 0,1 & 0,06 & 0,04 & DAE 314 \\
\hline Citrus sp. & Rutaceae & 1 & 0,06 & 0,01 & 0,1 & 0,06 & 0,04 & \\
\hline Guapira opposita (Vell.) Reitz & Nyctaginaceae & 1 & 0,06 & 0,01 & 0,1 & 0,06 & 0,04 & MCL 127 \\
\hline Cordia ecalyculata Vell. & Boraginaceae & 1 & 0,06 & 0,00 & 0,1 & 0,05 & 0,03 & DAE 287 \\
\hline $\begin{array}{l}\text { Plinia } \quad \text { trunciflora } \quad \text { (O.Berg) } \\
\text { Kausel }\end{array}$ & Myrtaceae & 1 & 0,06 & 0,00 & 0,1 & 0,05 & 0,03 & DAE 341 \\
\hline
\end{tabular}

componentes da vegetação nativa da Bacia do Rio Tibagi, duas outras espécies foram amostradas neste levantamento, Schizolobium parahyba (Vell.) Blake (guapuruvu) nativa da Mata Atlântica e Citrus sp. (laranja-do-mato), gênero nativo da região Indomalaia e da China (LÖTSCHERT e BEESE, 1994). A presença destas duas espécies pode ser explicada pela dispersão de indivíduos cultivados, já que o fragmento está localizado dentro de uma fazenda, na qual são desenvolvidas atividades agropecuárias nas últimas décadas.

Espécies como Trichilia clausseni (353 indivíduos), Actinostemon concolor (126 indivíduos), Sorocea bonplandii (99 indivíduos), Cabralea canjerana (74 indivíduos), Euterpe edulis (68), Nectandra megapotamica (65 indivíduos) e Guarea kunthiana (64 indivíduos) são as que possuem o maior número de indivíduos amostrados, representando juntas $54,11 \%$ do total de indivíduos. Vale destacar que a maioria dessas espécies são características de estágios finais na sucessão ecológica e estão presentes em remanescentes em bom estado de conservação.

Já as espécies Cordia ecalyculata, Crysophyllum marginatum, Bastardiopsis densiflora, Strychnos brasiliensis, Ruprechtia paranensis, Erythrina sp., Mollinedia clavigera, Myrsine laetevirens, Plinia trunciflora, Guapira hirsuta, Guapira opposita, Phytolacca dioica, Zanthoxylum rugosum, Guazuma ulmifolia e Aloysia virgata foram amostradas com apenas um indivíduo, representando $17,2 \%$ do total de espécies identificadas, e podem ser uma referência para a avaliação da riqueza de espécies do fragmento e justificar a necessidade de implementação de medidas para a sua conservação. A presença de poucas espécies com alta densidade e a maioria com poucos indivíduos é um padrão comum em florestas tropicais e subtropicais, e explica em parte a elevada diversidade destas florestas, pois não ocorre expressiva dominância ecológica entre as espécies (SILVA et al., 2012).

A espécie que mais se destacou em Valor de Importância foi Trichilia clausseni, resultado dos maiores valores de densidade relativa e frequência relativa, estando presente em $87 \%$ das parcelas amostradas. Esta espécie foi considerada por Viani et al. (2011) como exclusiva de FES, mas também está citada nos levantamentos de regiões de ecótono com a FOM.

Cabralea canjerana esteve presente em $49 \%$ das parcelas, resultando no segundo maior valor de frequência relativa (Tabela 1). Segundo Rodrigues et al. (2003), esta espécie é considerada de densidade variável e distribuição ampla dentro dos fragmentos florestais.

Actinostemon concolor também apresentou altos valores de densidade relativa e frequência relativa. Sendo uma espécie típica do sub-bosque das florestas ciliares (DIAS et al., 2002), obteve destaque neste levantamento talvez pelo fragmento estar em uma região limítrofe com o rio Tibagi, separado apenas por uma área de vegetação perturbada, resultante de atividades antrópicas anteriores.

Gallesia integrifolia (pau-d'alho) obteve expressivo valor em dominância relativa, por apresentar indivíduos de grande porte com os 
maiores valores de área basal $\left(12,28 \mathrm{~m}^{2} / \mathrm{ha}\right)$. Ficus guaranitica (figueira) apresentou o segundo maior valor em dominância relativa entre as espécies e, apesar de poucos indivíduos amostrados (quatro), destacou-se por apresentar indivíduos de grande porte, ocupando um valor alto de área basal $\left(6,13 \mathrm{~m}^{2} / \mathrm{ha}\right)$. A madeira das figueiras é usada na confecção manual de peças maciças, mas não é considerada nobre por ter resistência muito baixa (CARAUTA e DIAZ, 2002). Assim, a presença de indivíduos de grande porte de figueiras e paud'alho poderia ser explicada por estas espécies não terem sido extraídas seletivamente das matas, como outras espécies que apresentam madeira nobre $(A$. polyneuron e lauráceas).

Nectandra megapotamica, Cupania vernalis, Trichilia claussenii e Allophylus edulis foram consideradas as espécies mais características das FES do rebordo do planalto Meridional (RS) (KILCA e LONGHI, 2011) e praticamente todas elas se destacaram neste estudo, exceto Cupania vernalis que não foi amostrada.

Aspidosperma polyneuron, Cabralea canjerana, Euterpe edulis, espécies de Ficus, Gallesia integrifolia, Nectandra megapotamica e Syagrus romanzoffiana entre outras espécies, representaram o dossel ultrapassando os $15 \mathrm{~m}$ de altura. Segundo Dias et al. (2002), espécies emergentes como peroba, pau-d'alho e figueiras caracterizam as formações florestais do baixo Tibagi. Já o sub bosque (7-12 m) ficou representado por espécies como Actinostemon concolor, Guarea kunthiana, Picramnia ramiflora, Sorocea bonplandii e Trichilia clausseni.

Segundo Soares-Silva et al. (1992), Gallesia integrifolia (indicadora de fertilidade), Aspidosperma polyneuron, Astronium graveolens, Machaerium paraguariense, Balfourodendron riedelianum, Ocotea silvestris dentre outras, denotam com sua presença a riqueza de uma vegetação e estas espécies encontram-se bem representadas neste fragmento.

Euterpe edulis (palmiteiro) não foi encontrada na porção da mata mais próxima à estrada de acesso ao rio Tibagi, o que demonstra uma possível exploração a qual o palmiteiro pode ter sido submetido. Entretanto, mais distante da estrada, esta espécie foi amostrada em diferentes parcelas, o que explica sua posição entre as dez primeiras espécies ordenadas pelo IVI e o expressivo valor obtido de densidade relativa. O número de indivíduos encontrados (68) é suficiente segundo a pirâmide demográfica de uma população natural proposta por Reis et al. (2000), na qual no mínimo 61 indivíduos em estado reprodutivo podem manter populações naturais.

Achatocarpus pubescens, Astronium graveolens, Aspidosperma polyneuron, Lonchocarpus muelbergianus, Machaerium paraguariense e Myrocarpus frondosus estão na categoria de espécies raras, para o Estado do Paraná, listadas pela Secretaria do Meio Ambiente (1995), indicando a importância da conservação do fragmento. Essas espécies estão incluídas nesta categoria pela redução do seu habitat, mas ainda não estão em perigo de extinção. A. polyneuron também está presente na lista do IBAMA (Instrução Normativa $\mathrm{n}^{\circ}$. 06, de 23/09/2008).

$\mathrm{Na}$ análise de similaridade entre este fragmento e outros localizados em FES ou FES ecótono com FOM no estado do Paraná, foram encontradas um total de 451 espécies de árvores. As espécies Campomanesia xanthocarpa e Casearia sylvestris estiveram presentes em mais de $90 \%$ das localidades avaliadas. Já as espécies Aspidosperma polyneuron, Balfourodendron riedelianum, Cabralea canjerana, Holocalyx balansae, Ocotea puberula, Machaerium stipitatum, Nectandra megapotamica, Parapiptadenia rigida e Prunus myrtifolia foram amostradas em mais de $70 \%$ das localidades. Juntas essas espécies podem ser consideradas como típicas destas formações florestais FES ou FES ecótono FOM no estado do Paraná. Por outro lado, 36,14\% (163 espécies) foram amostradas em apenas uma localidade.

A Figura 1 mostra que dentre os levantamentos analisados, nove apresentaram baixa dissimilaridade, pois os valores do índice foram inferiores a 70. Entretanto, outros dez levantamentos apresentaram alta dissimilaridade, indicando certa heterogeneidade florística entre essas formações florestais no estado do Paraná. Expressiva similaridade florística entre FES e FOM foi constatado por Higuchi et al. (2013) quando estudaram o padrão fitogeográfico de florestas da região sul.

O grupo mais coeso foi representado por fragmentos localizados na região sudoeste do estado (Quedas do Iguaçu, Capitão Leônidas Marques e Dois Vizinhos), que, além da proximidade geográfica, são ecótonos de FES e FOM. Entre as espécies registradas para estas localidades, está a espécie símbolo da FOM, Araucaria angustifolia, que além de amostrada nestes locais, somente 


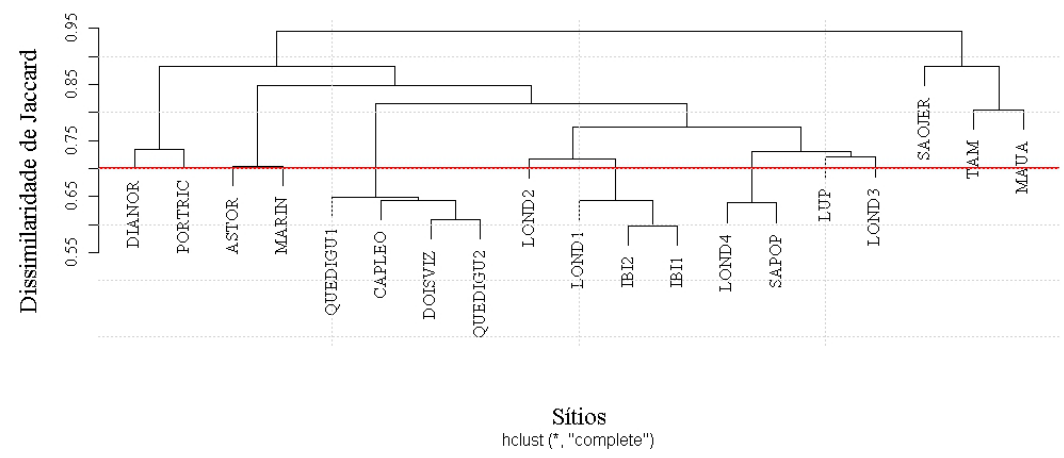

FIGURA 1: Dissimilaridade florística entre a área estudada e os demais levantamentos de vegetação no estado do Paraná. Agrupamento pelo método de ligação completa.

FIGURE 1: Floristic dissimilarity among the study site and other vegetation surveys in Parana state. Complete-linkage clustering.

foi encontrada em São Jerônimo da Serra, e espécies de Ilex, que foram amostradas quase que exclusivamente nessas áreas.

Um segundo agrupamento bastante similar foi formado por fragmentos localizados na região norte do estado, nos municípios próximos de Ibiporã, Londrina (incluindo o presente trabalho) e Sapopema, regiões típicas de FES. Algumas espécies ocorreram quase que exclusivamente nestes locais como Astronium graveolens, Euterpe edulis, Inga striata e Piptadenia gonoacantha.

Dois outros agrupamentos menores também podem ser associados com a proximidade geográfica e consequentemente com condições ambientais similares, foram eles, Diamante do Norte com Porto Rico, e Astorga com Maringá.

Os quatro levantamentos realizados em Londrina, sendo três deles na Mata dos Godoy, não se agruparam na análise como era esperado, tendo em vista que foram realizados no mesmo município e ou no mesmo local. Apesar da proximidade geográfica, a influência de cursos d'água deve ter sido um dos principais fatores ambientais determinantes na diferenciação florística destas áreas, tendo em vista, por exemplo, que o levantamento Lond3 (BIANCHINI et al., 2003) foi realizado somente em uma área alagável.

O agrupamento Mauá, Tamarana e São Jerônimo da Serra distingue-se dos demais, mas ficaram próximos devido à localização na interface do baixo com o médio Tibagi, com altitudes maiores e onde a vegetação pode ser considerada um dos ecótonos FES/FOM examinados. A flora mais distinta das demais foi São Jerônimo da
Serra, explicado pelo grande número de espécies exclusivas quase 25\% (38 spps.) Sua principal particularidade é incluir um enclave savânico, sendo que boa parte das espécies exclusivas são espécies típicas de cerrados do Paraná, como por exemplo, Anadenanthera peregrina (L.) Speg., Caryocar brasiliense Cambess, Couepia grandiflora (Mart. \& Zucc.) Benth. Ex Hook. f., Handroanthus ochraceus (Cham.) Mattos, Qualea cordata (Mart.) Spreng., Ouratea spectabilis (Mart. ex Engl.) Engl., Stryphnodendron adstringens (Mart.) Coville (RODERJAN et al, 2002).

A similaridade florística foi alta quando envolveu o mesmo tipo de unidade vegetacional e a proximidade espacial entre as áreas analisadas, conforme já constatado por Rodrigues e Nave (2000). A distância geográfica geralmente resulta em fatores ambientais diferenciados como clima, precipitação, condições geológicas, edáficas e topográficas, que influenciam na distribuição das espécies e atuam diretamente na similaridade florística entre as áreas (KUNZ et al., 2009). Higuchi et al. (2013) encontram em suas análises de padrões florísticos elevada substituição de espécies associadas principalmente à temperatura e à altitude. Por outro lado, localmente, as diferenças florísticas podem ser resultado de condições edáficas e topográficas, e do grau de perturbação do ambiente.

Outro fator importante nas análises de similaridade é que são utilizados diferentes trabalhos avaliados através de diferentes métodos de amostragem e critérios de inclusão, resultando em composições florísticas que nem sempre retratam a real riqueza e diversidade dos locais. 
No fragmento estudado, não foram observados locais com um maior grau de perturbação, indicados pela presença evidente de lianas e ou bambus. Tendo em vista que a fazenda é composta por diferentes fragmentos, seria interessante que fossem viabilizados corredores de ligação entre eles, possibilitando além do aumento no fluxo gênico entre as populações, a possibilidade de enriquecimento através da dispersão das espécies. No entanto, de extrema importância para a manutenção destas populações e dos processos ecológicos na RPPN, seria o rígido controle das atividades pecuárias da fazenda, impedindo o trânsito de gado nos fragmentos.

\section{CONCLUSÕES}

A análise da estrutura horizontal indicou que o maior fragmento da RPPN Mata do Barão encontra-se em estágio médio a avançado de conservação, com área basal por hectare alta, diversidade expressiva e riqueza de espécies em diferentes categorias sucessionais, inclusive com elevado número de indivíduos de espécies secundárias tardias ou clímax.

A proximidade geográfica associada a fatores ambientais semelhantes, assim como ao tipo de unidade fitogeográfica predominante, evidenciaram relações florísticas mais próximas, por outro lado, fatores ambientais locais como proximidade a cursos d'água diferenciaram a flora de locais próximos.

A área em estudo é um típico fragmento de Floresta Estacional Semidecidual na região Norte do estado do Paraná, e os resultados florísticos e de estrutura o caracterizam como de alto valor de conservação.

\section{AGRADECIMENTOS}

Aos técnicos da Universidade Estadual de Londrina pela ajuda em campo. À Escola Superior de Agricultura "Luiz de Queiroz" e aos responsáveis pela fazenda Figueira pela permissão do seu uso.

\section{REFERÊNCIAS BIBLIOGRÁFICAS}

APG III. An update of the Angiosperm Phylogeny Group classification for the orders and families of flowering plants: APG III. Botanical Journal of the Linnean Society, London, v. 161, n.2, p. 105-121, 2009.
BIANCHINI, E. et al. Diversidade e estrutura de espécies arbóreas em área alagável do município de Londrina, sul do Brasil. Acta Botânica Brasilica, v. 17, n. 3, p. 405-419, 2003.

CAMPOS, J.B.; SILVEIRA-FILHO, L. Floresta Estacional Semidecidual - Série Ecossistemas Paranaenses. Curitiba: SEMA, 2010. v. 5.

CARAUTA, J. P. P.; DIAZ, B. E. Figueiras no Brasil. Rio de Janeiro: Editora UFRJ, 2002.

COSTA, J.T. et al. Composição florística das espécies vasculares e caráter sucessional da flora arbórea de um fragmento de Floresta Estacional Semidecidual no sul do Brasil. Revista Brasileira de Botânica, v. 34, n. 3, p. 411-422, 2011.

DANNI-OLIVEIRA, I. M.; MENDONÇA, F.A. Dinâmica atmosférica e tipos climáticos predominantes da bacia do rio Tibagi. In: BIANCHINI, E. et al. A bacia do rio Tibagi. Londrina, 2002. p. 63-66.

DIAS, M.C. et al. Florística e fitossociologia das espécies arbóreas da bacia do rio Tibagi. In: BIANCHINI, E. et al. A bacia do rio Tibagi. Londrina, 2002. p. 109-124.

EMBRAPA - Empresa Brasileira de Pesquisa Agropecuária /IAPAR - Instituto Agronômico do Paraná. Levantamento de reconhecimento dos solos do estado do Paraná. Curitiba: EMBRAPASNLCS/Sudesul/IAPAR, 1984.

FERREIRA Jr., M. Comparação florística e estrutura do estrato arbóreo de dois fragmentos florestais na porção média da bacia do rio Tibagi, Paraná. 2009. Monografia (Graduação em Ciências Biológicas) - Universidade Estadual de Londrina, Londrina, 2009.

HIGUCHI, P. et al. Influência do clima e de rotas migratórias de espécies arbóreas sobre o padrão fitogeográfico de florestas na região sul do Brasil. Ciência Florestal, v. 23, n. 4, p. 539-553, 2013.

IBGE - Fundação Instituto Brasileiro de Geografia e Estatística. Manual técnico da vegetação brasileira. (Série Manuais Técnicos em Geociências n.1). Rio de Janeiro: FIBGE, 1992.

JUDD, W.S. et al. Sistemática Vegetal: Um enfoque filogenético. 3 ed. Porto Alegre: Artmed, 2009. $612 \mathrm{p}$.

KILCA, R.; LONGHI, S.J. A composição florística e a estrutura das florestas subtropicais estacionais nas escarpas do Planalto Meridional do Rio Grande do Sul In: SCHUMACHER et al. (Orgs.). Floresta Estacional Subtropical: Caracterização e Ecologia nas Escarpas da Serra Geral. Santa Maria: UFSM, 2011. p. 53-83. 
KILMA, L. et al. Levantamento da vegetação arbórea dos remanescentes naturais da Araupel S/A, Quedas do Iguaçu, PR.. In: IV CONGRESSO FLORESTAL PARANAENSE, 2012, Curitiba. Anais... 2012.

KREBS, C. J. Ecological Methodology. New York: Addison Wesley Longman, 1999.

KUNZ, S.H. et al. Análise da similaridade florística entre florestas do Alto Rio Xingu, da Bacia Amazônica e do Planalto Central. Revista Brasileira de Botânica, v. 32, n. 4, p. 725-736, out./ dez. 2009.

LEITÃO FILHO, H.F. Considerações sobre a florística de florestas tropicais e subtropicais do Brasil. IPEF, v. 35, p. 41-46. 1987.

LÖTSCHERT, W.; BEESE, G. Tropical plants. Collins photo guide. London: Harper Collins Publishers, 1994.

LOUREIRO, W. A situação atual do sistema de unidades de conservação do Paraná. In: CONGRESSO BRASILEIRO DE UNIDADES DE CONSERVAÇÃO, 4, 2004. Curitiba. Anais... Curitiba: Fundação $\mathrm{O}$ Boticário de proteção à Natureza e Rede Pró-unidades de Conservação, 2004, p. 135-148.

MACHADO, E.L.M. et al. Efeitos do substrato, borda e proximidade espacial na estrutura da comunidade arbórea de um fragmento florestal em Lavras, MG. Revista Brasileira de Botânica, v. 31.n. 2, p. 287-302. 2008.

MAGURRAN, A.E. Ecological diversity and its measurement. New Jersey: Princeton University Press, 1988.

MARESTONI, T.M. Diversidade e Estrutura das Espécies arbóreas na Reserva Particular do Patrimônio Natural Mata São Pedro, Lupionópolis - Paraná. 2007. Trabalho de Conclusão de Curso (Curso de Ciências Biológicas) - Universidade Estadual do Norte do Paraná, Campus Luiz Meneghel, Bandeirantes.

MARTINS, F. R. Estrutura de uma floresta mesófila. 2. ed. Campinas: Editora da Unicamp, 1993.

MORESCHI, J.; SAMPAIO, A.C.F. Análise florestal e das condições de sucessão vegetal para subsidiar enriquecimento florestal em fragmento do Parque dos Pioneiros, Maringá, Paraná, Brasil. Revista em Agronegócios e Meio Ambiente, v.1, n.3, p. 309-325, 2008.

OLIVEIRA-FILHO, A.T.; FONTES, M.L. 2000. Patterns of Floristic Differentiation among Atlantic Forests in Southeastern Brazil and the Influence of Climate. Biotropica, v. 32, n. 4(b), p. 793-810, 2000.

PEZZATTO, A. W. Composição florística e ciclagem de macronutrientes em diferentes seres sucessionais nas margens de reservatório de Hidrelétrica no oeste do Paraná. 2004. Dissertação (Mestrado em Ciências Florestais, Curso de PósGraduação em Engenharia Florestal, Setor de Ciências Agrárias), Universidade Federal do Paraná, Curitiba.

PINESE, J. P. P. Síntese Geológica da Bacia do Rio Tibagi. In: BIANCHINI, E. et al. A bacia do rio Tibagi. Londrina, 2002.

$\mathrm{R}$ Development Core Team. R: A language and environment for statistical computing. Vienna, Austria: R Foundation for Statistical Computing, 2010.

REIS, M.S. et al. Management and Conservation of Natural Populations in Atlantic Rain Forest: The Case Study of Palm Heart (Euterpe edulis Martius). Biotropica, v. 32, n. 4(b), p. 894-902, 2000.

RODERJAN, C.V. et al. As unidades fitogeográficas do Estado do Paraná. Ciência \& Ambiente, v. 24. p.75-92, 2002.

RODRIGUES, L.A. et al. Florística e estrutura da comunidade arbórea de um fragmento florestal em Luminárias, MG. Acta Botânica Brasilica, v. 17, n. 1, p. 71-87, 2003.

RODRIGUES, R.R.; NAVE, A.G. 2000. Heterogeneidade florística das matas ciliares. In: RODRIGUES, R.R.; LEITÃO FILHO, H.F. (eds.). Matas ciliares: conservação e recuperação. São Paulo: Editora da Universidade de São Paulo, 2000. p. 45-71.

ROSSETTO, E.F.S., VIEIRA, A.O.S. Vascular flora of the Mata dos Godoy State Park, Londrina, Paraná, Brazil. Check list and Authors, v. 9, n. 5, p. 1020-1034, 2013.

SÁ, K.L.V.R. A Flórula Vascular da Reserva Indígena São Jerônimo, São Jerônimo da Serra- Paraná: subsídios para conservação da vegetação. 2004. 126p. Dissertação (Mestrado em Biologia Vegetal) - Universidade Estadual de Campinas. Campinas.

SAKURAGUI, C.M.; JANUNZZI, A.M. Flora Fanerogâmica. In: BENEDITO, E. Ecologia do Ribeirão Diamante, Estação Ecológica do Caiuá, PR. Maringá: Eduem, 2009. p. 27-40.

SECRETARIA DO MEIO AMBIENTE. Lista Vermelha de Plantas Ameaçadas de Extinção no Estado do Paraná. Curitiba: SEMA/ 6TZ, 1995. SILVA, A.C. et al. Relações florística e 
fitossociologia de uma Floresta Ombrófila Mista Montana secundária em Lages, Santa Catarina. Ciência Florestal, v. 22, n. 1, p. 193-206, 2012.

SILVA, F.C. et al. Composição florística e fitossociologia do componente arbóreo das florestas ciliares da bacia do rio Tibabi.3.Fazenda Bom Sucesso, município de Sapopema, PR. Acta Botânica Brasilica, v. 9, n. 2, p. 289-302, 1995.

SOARES-SILVA, L. H. et al. Composição florística e fitossociológica do componente arbóreo das florestas ciliares da bacia do rio Tibagi: Fazenda Doralice- Ibiporã, PR. In: CONGRESSO NACIONAL SOBRE ESSÊNCIAS NATIVAS, 2, 1992, Anais..., p. 199-206.

SOARES-SILVA, L. H. et al. Fitossociologia de um trecho de floresta de galeria no Parque Estadual Mata dos Godoy, Londrina, PR, Brasil. Bol. Herb. Ezechias Paulo Heringer, v. 3, p. 46-62, 1998.

SOUZA, D. R. et al. Levantamento Florístico e chave de identificação através de caracteres vegetativos das espécies da mata remanescente do Campus da UTFPR de Dois Vizinhos, PR. In: I SEMINÁRIO DE EXTENSÃO E INOVAÇÃO DA UTFPR - SEI, 2011, Curitiba. Anais... Curitiba, 2011.

SOUZA, M.C.; MONTEIRO, R. Levantamento florístico em remanescente de floresta ripária no alto rio Paraná: Mata do Araldo, Porto Rico, Paraná, Brasil. Acta Scientiarum, v. 4, n. 27, p. 405-414, 2005.

STIPP, N. A. F. 2002. O rio Tibagi no contexto hidrogeográfico paranaense. In: BIANCHINI, E. et al. A bacia do rio Tibagi. Londrina, 2002. p. 39-43. VEIGA, M.P. et al. Avaliação dos aspectos florísticos de uma mata ciliar no Norte do Estado do Paraná. Acta Scientiarum Agronomy, v. 25, n. 2, p. 519-525, 2003.

VIANI, R.A.G. et al. Caracterização florística e estrutural de remanescentes florestais de Quedas do Iguaçu, Sudoeste do Paraná. Biota Neotropica, v. 11, n. 1, p. 115-127, 2011. 